\title{
Development of a Graphic Information System Applied to Quality Statistic Control in Production Processes
}

\author{
Laura Vázquez ${ }^{1}$, Alicia Valdez ${ }^{2}$, Griselda Cortes ${ }^{3}$, Mariana Rosales ${ }^{4}$ \\ Research Center Autonomous University of Coahuila \\ Coahuila, Mexico
}

\begin{abstract}
One of the advantages that organizations have when using an Information System is the control of their activities. This article develops an Information System that will allow an organization to graphically obtain the real results of a production process by applying Nelson's eight rules to determine if any measured variable is out of control. The software architecture pattern used is the Model View Controller (MVC) to keep the functionality of the application separate. The front-end, that is, the part that interacts with the users, was developed in ASP.NET as a web platform to provide the required services, JavaScript, HTML 5, Razor and Bootstrap. The back-end, which is the part that processes the entry of the front-end and performs the calculations, operations, communication with the database and reading of files, was developed with the $\mathrm{C}$ sharp programming language, the SQL Server database management system and the entity framework. As a result, the system sends an e-mail as an alarm with an explanation of what has happened when it detects that some measured variable is out of control by applying Nelson's rules. This allows the organization to make effective decisions in the processes involved.
\end{abstract}

Keywords-Information system; Nelson's rules; Model View Controller pattern; C\#; ASP.NET

\section{INTRODUCTION}

Technology can help all kinds of businesses improve the efficiency and effectiveness of their business processes, managerial decision making, and workgroup collaboration, which strengthens their competitive positions in rapidly changing marketplaces [1].

Also, technology is used to solve problems, through its systems that must be adapted to the needs of the organizations.

Information Systems and Technologies are vital components of successful businesses and organizations some would say they are business imperative [1]. Information System is defined as group of elements organized with the purpose of supporting management and operational decision making [2].

It is important to mention that Information Systems can be developed using fourth-generation software tools; their functionality is that users in organizations can access data, create, and interpret reports quickly to facilitate processes.

The benefits that an organization has when using an Information System in the long term are the following: automation of operational processes, provision of an information platform for decision making, and achievement of competitive advantage.

In the production processes, different factors and elements are combined on which measurements must be made, that is to say, in each process it is necessary to control variables such as pressure, weight, flow, etc. to guarantee the quality of a product.

According to the above, it is necessary to develop a system as a support tool that allows us to obtain graphically the real results of a productive process, applying Nelson's eight rules. As it will be explained later, these rules will allow controlling the production process to determine if any measured variable is out of control.

The theoretical fundamental for the development of the graphic Information System is shown below, considering the MVC software architecture model and the software used for the back-end and front-end. The results expected at the end of the processing as output are directly related to the characteristics and processing development of the proposed Information System, which guarantees the efficiency of its effectiveness.

\section{RELATED WORKS}

For the area of education, the work in [3] presented the Development of Information System for a University.

The student Information System of a university stores and tracks all student data which are needed by the faculty and staff to manage the operations of the university. Information such as grades, attendance records, admission information, and financial aid are tracked through these platforms [3].

In this paper [3], students Information System has been developed to maintain the information and other content of digitized Information using ADO .NET technology and Microsoft SQL. This system is mainly intended to be used by the staff from the student affairs department and faculties of the university.

In the paper titled "Implementation of Scrum work framework in the development of quality assurance Information System" uses the Scrum agile development methodology.

The purpose of this research is to develop a quality assurance Information System by implementing the Scrum 
Framework. Scrum is one of the popular frameworks in Agile Development Methodology. In this way, the development of productivity increases significantly. In this Applied Research, the Action Research approach is used [4].

The work in [5] presented a system that was designed using PHP and MySQL as the programming language for the database. The system can classify the brown sugar by calculating the weight of the criterion. Besides, the classification process is performed to determine the optimal value.

\section{THEORICAL FUNDAMENTAL}

\section{A. Information System}

An Information System can be any organized combination of people, hardware, software, communications networks, data resources, and policies and procedures that stores, retrieves, transforms, and disseminates information in an organization [1].

The requirements of an Information System are determined by the objectives of the organization for which the system is being designed and built [6].

The functions of Information Systems according the reference [2] are the following:

- As a source of information to help in effective decision making by managers.

- A contributor to productivity efficiency and customer satisfaction.

- The Information System is useful to achieve success in various functions such as Finance, operations, marketing, human resource, and store management.

In addition, Bagad in his book "Managenent Information Systems" [2] comments on Information System Activities. It is indicated that in a business process, the different information processing activities take place. For example:

1) The input of data resources

2) Processing of data into information

3) The output of information products

4) Storage of data resources

5) Control of system performance

\section{B. Nelson's Rules}

The Nelson rules were published first in the October 1984 issue of the Journal of Quality Technology in an article by Lloyd S. Nelson. Nelson rules are methods in process control of determining if some measured variable is out of control [7].

These zones are used in conjunction with a set of pattern analysis rules to determine when a process has gone out of control [9]. In general, when identifying these rules, the region between the usual \pm 3 sigma limits are divided into six region and the pattern is explained with respect to $\pm 1,2$ and 3 sigma limits as shown in the Fig. 1 [8].

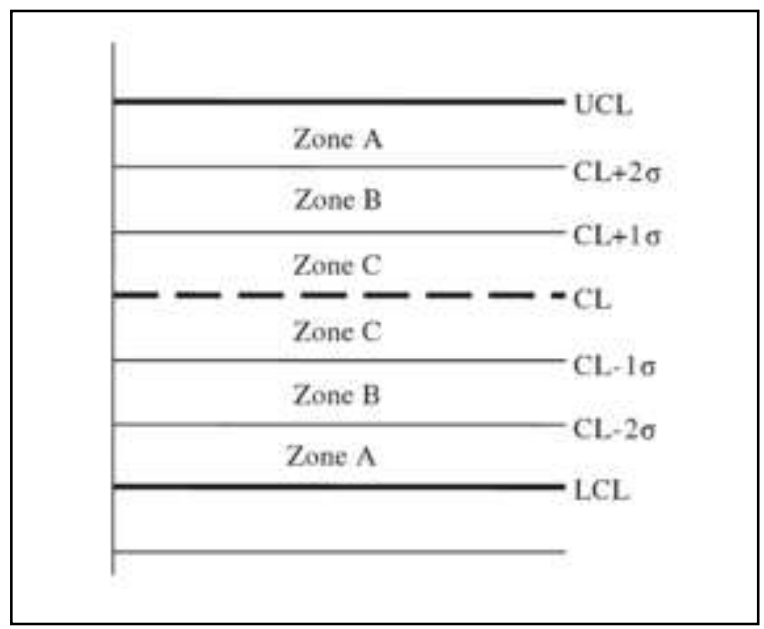

Fig. 1. Zones in a Control Chart. Source [8].

Nelson's rules shown in his 1984 article [7] are as follows:

- Rule 1. One point is more than three standard deviations from the mean.

- Rule 2. Nine (or more) points in a row are on the same side of the mean.

- Rule 3. Six (or more) points in a row are continually increasing (or decreasing).

- Rule 4. Fourteen (or more) points in a row alternate in direction, increasing then decreasing.

- Rule 5. Two (or three) out of three points in a row are more than two standard deviations from the mean in the same direction.

- Rule 6. Four (or five) out of five points in a row are more than one standard deviation from the mean in the same direction.

- Rule 7. Fifteen points in a row are all within one standard deviation of the mean on either side.

- Rule 8. Eight points in a row exist, but none within one standard deviation of the mean, and the points are in both directions from it.

The rules apply to an XS control chart in which the magnitude of some variable is plotted against time. The rules are based on the mean value and standard deviation of the samples considered through time.

\section{Software Development Tools}

1) Integrated Development Environment

IDE support means the tools can generate code, help you write code, and provide features and artifacts that accelerate your coding. Here is where many third-party languages often fall short. It takes a lot to provide IDE support to build the many application types Visual Studio enables [10]. Visual Studio is part of the family of integrated development environments (IDE) [11]. 
2) Programming Language

Visual $\mathrm{C \#}$ is a programming language designed for those who are familiar and comfortable programming in C-Style languages (such as C, C++, and Java). C\# is type -safe, objectoriented, and targeted for rapid application development. C\# developers tend to spend more of their time inside the Visual Studio code editor and less time with the designers [10].

ASP.NET is a web platform that provides all the services that you require to build enterprise-class server-based Web applications. One of the major benefits of ASP.NET is the change from interpreted code, previously used for Classic ASP (the programming model before ASP.NET), to compiled code, allowing web application to have better performance [12].

In addition, ASP.NET includes the following features [12]:

- A page and controls frameworks

- The ASP.NET compiler

- Security infrastructure

- Application configuration

- Health monitoring and performance features

- Debugging support

Razor is a template syntax that allows you to combine code and content in a fluid and expressive manner. Razor lets you write code using languages such as C\# or Visual Basic.NET [13].

ASP.NET Razor uses a simple programming syntax that lets you embed server-based code into a web page [12].

ASP.NET MVC is a free fully supported framework for building web applications that use the model-view-controller pattern. The MVC pattern itself makes it easier to manage complexity by clearly separating the functionality of the application into three core parts, the model, the view, and the controller [12].

Fig. 2 shows a simple implementation of the MVC pattern.

The straight arrows indicate direct associations, whereas curved arrows identify indirect associations.

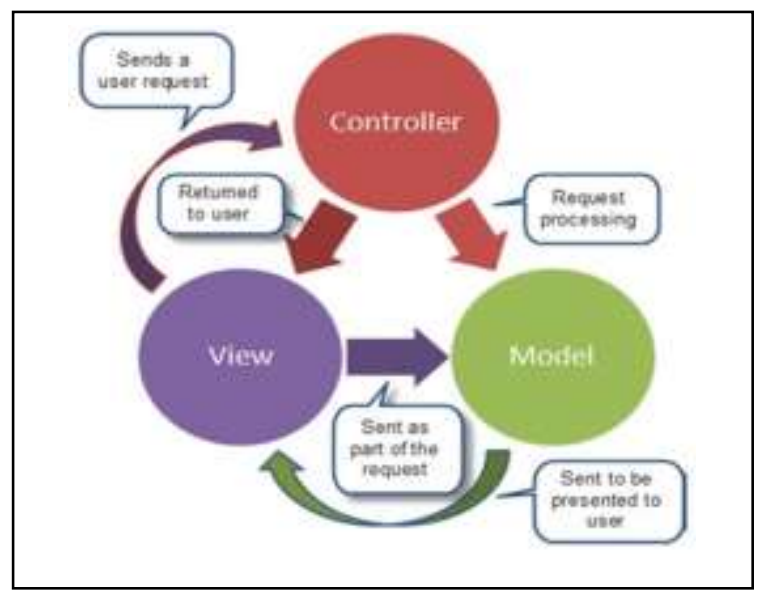

Fig. 2. Model-view-Controller Implementation. Source [12].
Explanation of Fig. 2 is shown below:

Models: models are basically a collection of classes where you will be working with data logic as well as business logic. Views: views are nothing but a pure HTML which decides how the UI (User Interface) is going to look like. Controller: Controllers are basically a bunch of classes which handles communication from the user [14].

ASP.NET MVC works well for web applications that are supported by large teams of developers and for web designers who need a high degree of control over the HTML [12]. ASP.NET is a complete and effective low-cost answer. It allows processes to be separated effectively continued with its effectiveness. This is done through the MVC solution. The ease of maintenance and scalability of ASP.NET depends on the skills of the developer. If the developer has enough programming knowledge, in the development of $\mathrm{C \#}$, the framework will be simple to use and take advantage of its main characteristics.

JavaScript is a programming language, light, interpreted, object-oriented, prototype-based and first-class functions, better known as the Web's scripting language. It is the programming language that Netscape created to bring your browser to life (on the front end client side) [15]. JavaScript is a scripting language that allows you to create dynamically updated content, control multimedia, animate images, and just about everything else.

The main goal of Bootstrap is to provide a web frontend framework for responsive developing with cross-browser compatibility [16]. Bootstrap is an open source product. It has evolved from being an entirely CSS-driven project to include a host of JavaScript plugins and icons that go hand in hand with forms and buttons [17].

One of the highlights is the build took on Bootstrap's website, where you can customize the build to suit your needs, choosing which CSS and JavaScript features you want to include on your site [17].

CSS is the recommended format for pages written in HTML based on "Cascading Style Sheets" standards. CSS allows the use of methods to create structures by treating the styles separately, reducing the rendering time, offering the client a faster and more efficient connection.

Also, HTML documents using CSS are small, since the style design is used. Therefore, the page increases the speed of transmission of content information, benefiting customers, page owners, and Web server administrators.

SQL Server is an enterprise-class database management system (DBMS) that is capable of running anything from a personal database only a few megabytes in size on a handheld Windows Mobile device up to a multiserver database system managing terabytes of information [18].

The database is effectively the highest-level object that you can refer to within a given SQL Server [19]. Besides, SQL Server offers a variety of administrative tools to ease the burdens of database development, maintenance, and administration. 


\section{METHODOLOGY}

Fig. 3 shows the diagram of the Information System developed.

The MVC software design pattern was used in the development of the system. The part of the front-end, that is, the one that interacts with the users was developed in ASP.NET, JavaScript, HTML 5, Razor and Bootstrap. On the other hand, for the back-end, which is the part that processes the input from the front-end and performs the calculations, operations, communication with the database and file reading, it was developed with C\#, SQL Server and entity framework, as described.

ASP.NET is the development environment used for the creation of the web page, using $\mathrm{C \#}$ as the back-end.

Fig. 4 shows the design of the controller view model of the Information System developed.

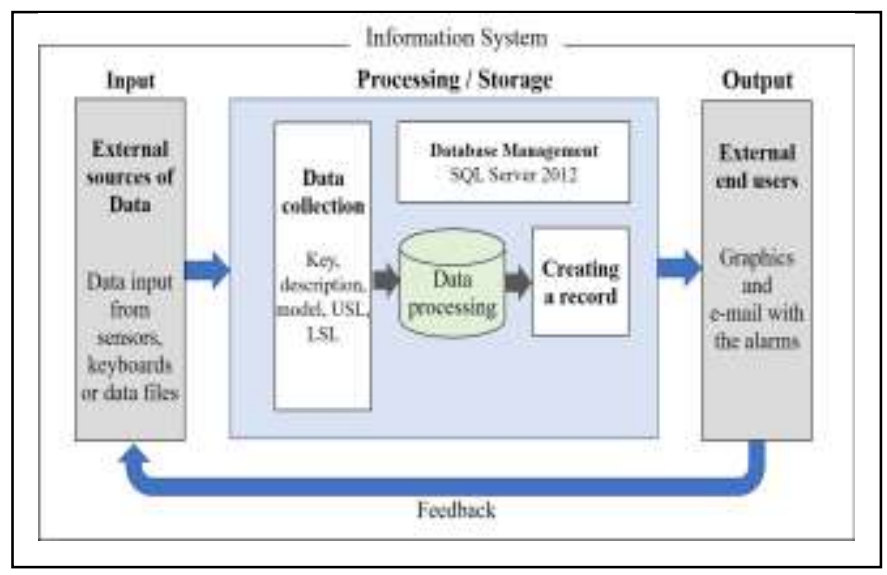

Fig. 3. Diagram of the Graphic Information System.

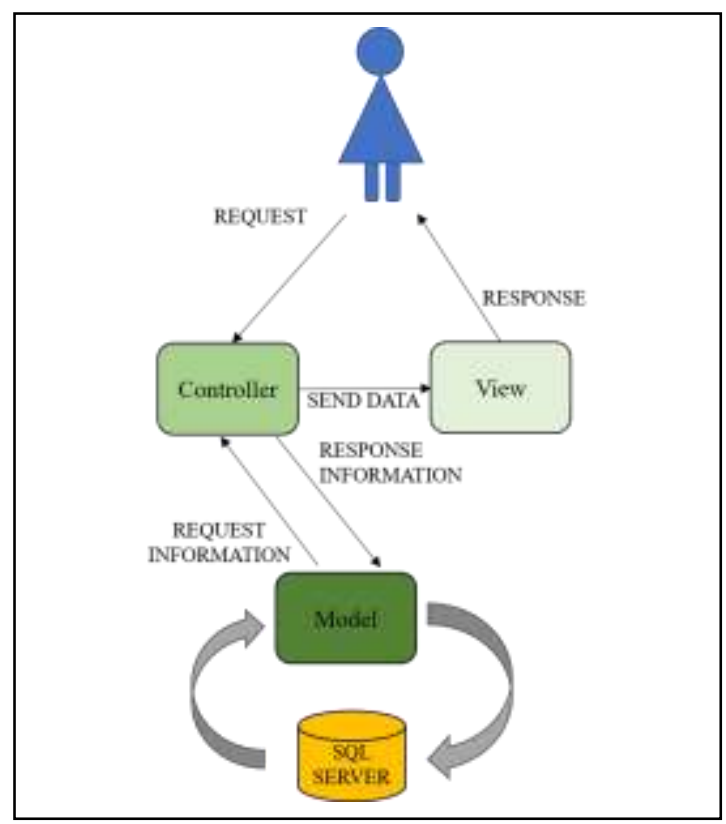

Fig. 4. Model Vista Controller Pattern Designed for the System.
In ASP.NET a web server was created with the style of MVC software architecture. When the client tries to navigate to a specific URL, the URL is taken by the controller, it uses the models (classes) and returns a view that is the page shown to the client.

To create this page, environments and languages were used such as HTML 5 to create the graphics, JavaScript to perform calculations on the client-side, Bootstrap and CSS for the design part (fonts and colors).

For the back-end, C\# was used to communicate with databases in SQL Server 2012 and to perform calculations. It is significant to mention that this database management system was used because ASP.Net has stable communication with SQL Server and offers access to .net functionalities.

Fig. 5 shows the tables used in the developed system.

As mentioned above, one element that Information Systems must have is data entry. In the system designed the ways to make the data entries are the sensors, the keyboard or from data files.

To start working in the system, the corresponding user must capture the information requested in the window shown in Fig. 6.

This information corresponds to the configuration of the variables of the production processes that will be subject to measurement. The data are headed as follows: key, description, model, upper specification limit (USL) and lower specification limit (LSL), and will form the records in the database used.

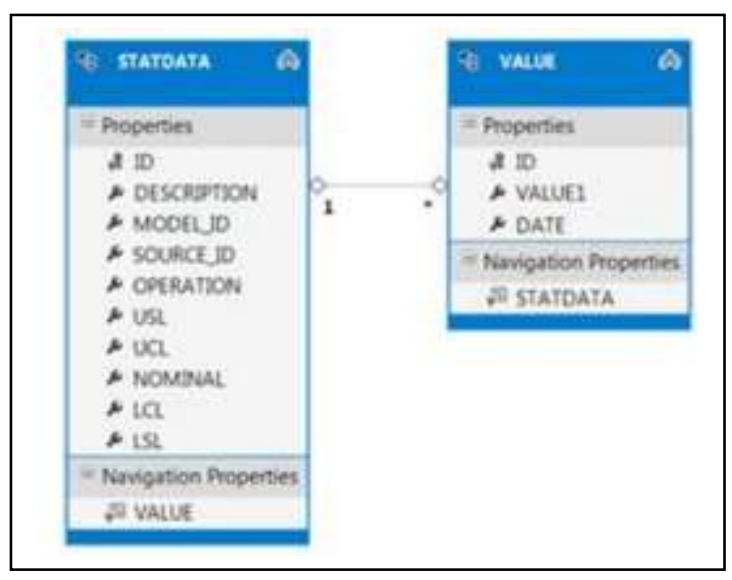

Fig. 5. System Tables.

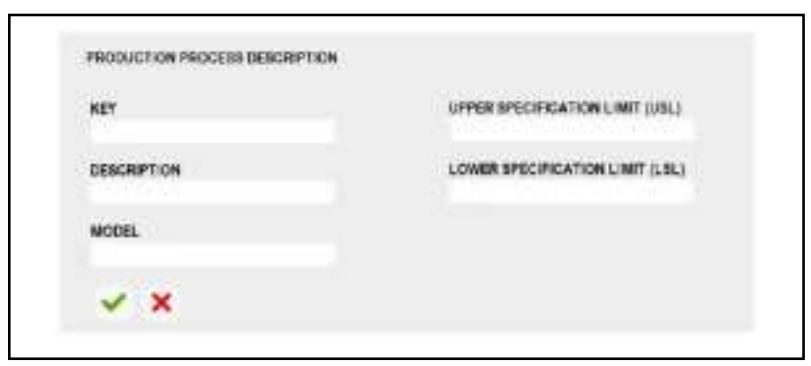

Fig. 6. Configuration Data Recording. 
Once the system has the necessary data to work, such as: the keys, the description, the model, the USL and the LSL, form the data collection that will be processed and stored. This information corresponds to the variables that will be measured in the production processes of the organization. Nelson's eight rules will apply to these variables for their corresponding evaluation.

The variables considered for evaluation are determined by the productive processes of each organization.

Fig. 7 shows some of the records captured in the system. The data considered are the following: total weight, total volume, humidity test, noise level, resistance, temperature, etc. In this same window, it is possible to make some changes in the data of the records.

The above information will be stored in the database of the SQL Server 2012 management system. Once the information is processed, the system will show the data output graphically, as it will be explained later.

Fig. 8 shows the data captured in the system, which corresponds to the measured variable "weight" of a production process.

The system considers the observations of the sample made, calculates the arithmetic mean and the standard deviation of the set of results. It also uses the established factors to build control diagrams, applying the corresponding coefficients to calculate the graph control limits in the formulas allowing the creation of XS control charts.

\begin{tabular}{|c|c|c|c|c|c|}
\hline Mer & DESCRYTION & WOOE & U\%: & Lst & exalied \\
\hline 4621 & TOTAL WEOHT & EDSW-1ase? & 30 & 238 & * \\
\hline 2514 & TOTAL VOLUME & 1AERS-29:4 & 470 & as & ne \\
\hline 3652 & NETRL, DETECHCN JEST & 78ERF-68650 & 25 & 20 & an \\
\hline 1634 & HUUOTI TEST & ABNBO-147 & 120 & $\mathrm{t17}$ & nu \\
\hline t667 & Nos: LeVEL & 2uassient & st & ss & $\mathbf{n}=0$ \\
\hline 3625 & REsistanCE & J1LKS-7Se & म & is & $\Delta=0$ \\
\hline zBs4 & TEMFEHATURE & RLow-J2z & 3 & 60 & $\mathbf{n e}$ \\
\hline 2502 & VEHIFICATICN of ERMMETR & $25 v 56-364$ & 544 & 541 & $x=0$ \\
\hline
\end{tabular}

Fig. 7. Data Storage.

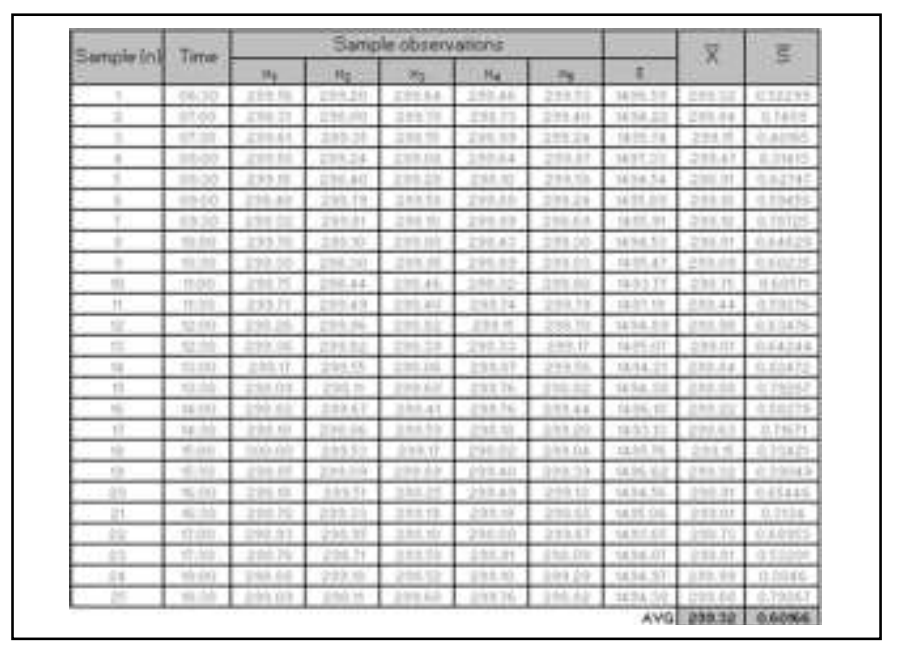

Fig. 8. Calculations made by the Information System.

\section{RESUlts}

Once the configuration of the corresponding parameters is done, and the data entered in the Information System completes, the system makes the control charts to analyze and determine if any variable is out of control.

Nelson's eight rules apply to each of the variables involved in the production process. The magnitude of the variable gets plotted against time. As mentioned above, the rules bases on the arithmetic mean and standard deviation of the samples.

As a result, the developed Information System performs the processing and analysis of the variables and displays the XS control chart. Fig. 9 shows the XS control graph corresponding to the measured variable "weight", where the real results of the arithmetic mean applied to the samples are observed. In addition, the upper and lower control limits and the additional 1 and 2 sigma limits are plotted on the control chart, as shown.

Fig. 10 shows the XS control chart of the previous variable, but with the standard deviation data calculated. The control limits and the 1 and 2 sigma limits are the same.

Remembering Nelson's Rule 6 which says: Four (or five) out of five points in a row are more than one standard deviation from the mean in the same direction [7], as shown in Fig. 11.

As can be seen, in the graph in Fig. 9, Nelson's rule number 6 is presented, since samples $5,6,7,8$, and 9, which are points in a row, are more than one standard deviation from the mean in the same direction, as shown in Fig. 12.

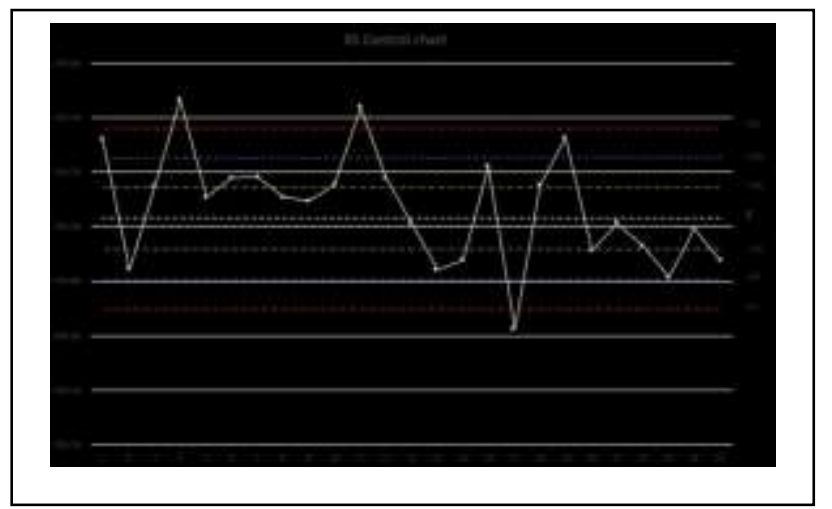

Fig. 9. XS Control Chart of the Arithmetic means of the Calculated Results.

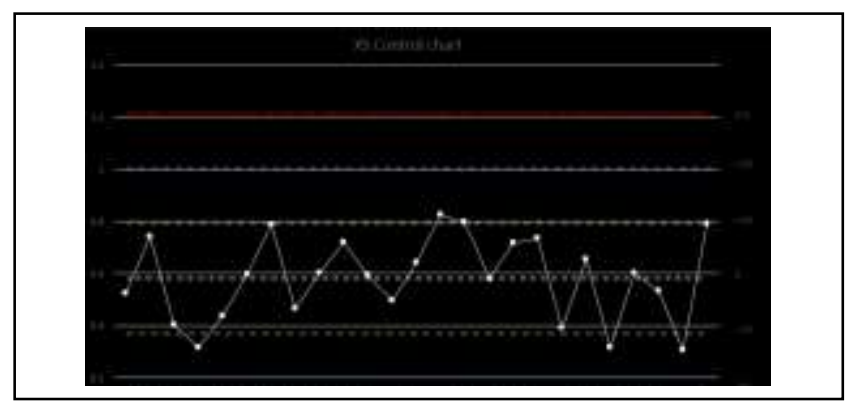

Fig. 10. XS Control Chart of the Standard Deviation of the Calculated Results. 


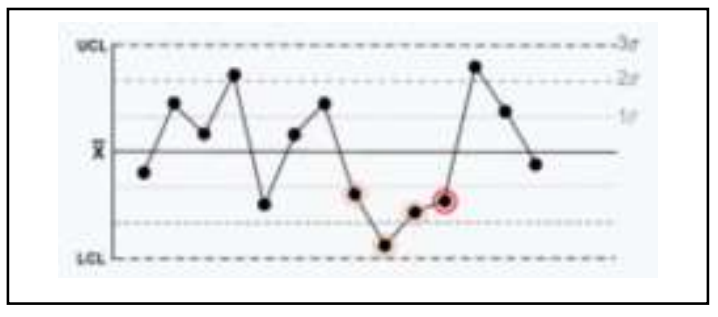

Fig. 11. Nelson's Rule 6.

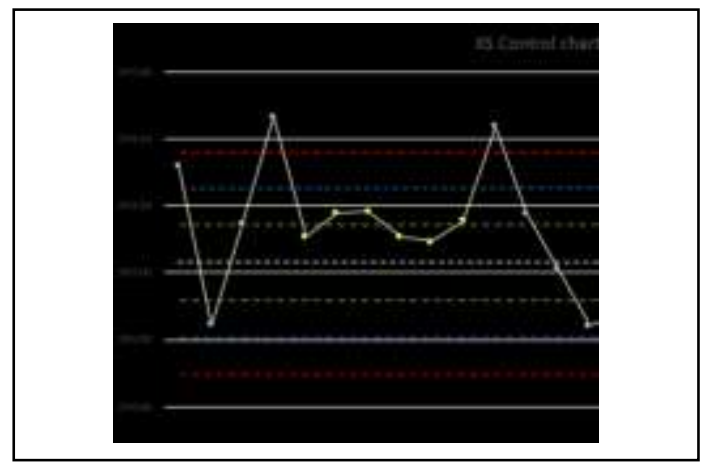

Fig. 12. First Segment where Nelson's Rule 6 was Detected.

At another point in the same graph, the Information System detects Nelson's Rule 6, as shown in Fig. 13 and displays it as a result in the alarm, as can be seen in Fig. 14.

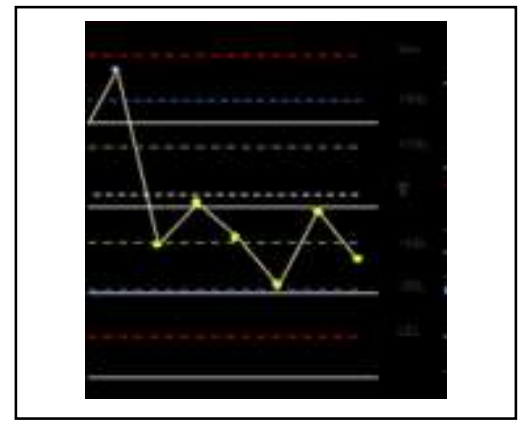

Fig. 13. Second Segment where Nelson's Rule 6 is Detected.

The Information System shows as a result once the data analysis is done and graphed, an e-mail with the alarms that have occurred when applying Nelson's eight rules and detecting if any measured variable is out of control, as shown in the Fig. 14. In this case, the variable measured was the total weight that has a KEY 4421.

As explained above, Nelson's rules are a set of methods that detect trends in the analyzed data. The system also shows the following values: Upper Specification Limit (USL) and Lower Specification Limit (LSL), as well as the automatically calculated values: Upper and Lower Control Limits, average and standard deviation.

\section{TOTAL. WEIGHT (4421) Alert.}

- Four (or five) out of five points in a row are more than 1 standard deviation from the mean in the same direction.

Fig. 14. Alarm Presented by the Information System.
The developed Information System allows to analyze in a graphic way the real measures in the productive processes, facilitating the decision making in real time by issuing the corresponding alarms.

\section{CONCLUSION}

As a conclusion, the Information Systems allows the organization to have effective control over activities. In addition, such systems help to increase the effectiveness in the productive processes, since they anticipate and foresee situations so that the decision making is effective.

Nelson's rules allow us to know and analyze the variables that are measured in the production processes and that are out of control, improving the quality of the products.

The use of these rules facilitates the interpretation of the control charts, but the experience of the human expert should indicate to what extent the results should be taken.

A trend in Information Systems is the use of technology as an essential part of corporate strategy, achieving an administrative advantage by facilitating decision making. In addition, technology is important for design and production control.

The use of technology will transform the organization, changing its structure. The information technology will significantly support the redesign of the processes through the Information Systems.

It is important to mention that the future work for the application and development of this paper could be to develop usable systems to facilitate the monitoring of process quality from the design of a product until it leaves the factory and is delivered to the final customer. On the other hand, it can be used in complementary areas for quality improvement in production processes, such as sales, inventory and purchases.

\section{VII.DISCUSSION}

With the development of Science and technology, the Information System is essential in our daily life, according to the reference [3].

The use of Information Systems will continue to be useful and effective in decision-making in various areas of each organization.

Organizations should use a system that ensures that their manufactured products meet specified quality standards, keeping in mind that the system must be tailored to the needs of that organization.

The author in [4] indicates an important factor that must be taken into account for the successful development of the Information System proposed in its research: human resources management, that is, the importance of the composition of the staff teams involved.

REFERENCES

[1] R. Behl, J. A. O’Brien and G. M. Marakas, Management Information Systems, 11th ed. India: Mc GrawHill Education.

[2] V. S. Bagad, Managenent Information Systems, 4th ed. Pune: Technical Publications Pune, 2009, pp. 1,12. 
[3] T. Htwe and M. Phyo Aung, "Development of Information System for a University", International Journal of Scientific and Research Publications, vol. 9, no. 6, pp. 494-499, June 2019, doi: 10.29322/IJSRP.9.06.2019.p9071.

[4] M. Broto, B. Indiarto and D. Prayitno, "Implementation of Scrum work framework in the development of quality assurance Information System", Jurnal Penelitian Pos dan Informatika, vol. 9, no. 2, pp. 125139, November 2019, doi: 10.17933/jppi.2019.090204.

[5] I. Taufik, A. Saleh, C. Slamet, D. S. Maylawati, M. A. Ramdhani and B. A. Muhammad, "Decision support system design for determining brown sugar quality with weighted product method", Journal of Physics: Conference Series, vol. 1280, no. 2, pp. 1-8, doi:10.1088/17426596/1280/2/022019.

[6] A. Olivé, Conceptual Modeling of Information Systems. Berlin: Springer, 2007, pp. 2.

[7] L. S. Nelson, "The Shewhart Control Chart - Tests for Special Causes", Journal of Quality Technology, 16 no. 4, pp. 237-239, October 1984.

[8] C. Kahraman and S. Yanik, Intelligent Decision Making in Quality Management: Theory and Applications. Switzarland: Springer, 2016, pp. 41-42.

[9] S. Boslaugh, Statistics in a Nutshell, 2nd ed. United States of America: O’Reilly, 2012, p.p. 345.
[10] M. Snell and L. Powers, Microsoft Visual Studio 2012 Unleashed, 1st ed. Indiana: Pearson Edication, Inc., 2012.

[11] B. S. Guérin, ASP.NET en C\# con Visual Studio 2015: Diseño y desarrollo de aplicaciones Web. Barcelona: Ediciones ENI, 2016, pp. 18.

[12] J. R. Guay, Beginning ASP.NET MVC 4, The exprert's voice in .NET. Apress, pp. 1,2, 6-9.

[13] J. Chadwick, Programming Razor: Tools for Templates in ASP.NET MVC or WebMatrix, 1st ed. United States of America: O’Reilly, 2011, pp. 3.

[14] R. Sahay, Hands on with ASP.NET MVC: Covering MVC 6, Quills Ink Publishing, 2014.

[15] A. A. Castillo, Curso de Programación Web: JavaScript, Ajax y jQuery, 2nd ed. ITCampus Academy, 2017, pp. 13-14.

[16] S. Moreto, M. Lambert, B. Jakobus and J. Marah, Bootstrap 4 Responsive Web Design, Birmingham: Packt Publishing, 2016, pp. 1.

[17] J. Spurlock, Bootstrap: Responsive Web Development, 1st ed. United States of America: O’Reilly, 2013.

[18] R. Rankins, P. Bertucci, C. Gallelli and A. T. Silverstein, Microsoft SQL Server 2012 Unleashed, 2nd ed. United States of America: Pearson Education, Inc., 2014, pp. 9.

[19] P. Atkinson and R. Vieira, Beginning Microsoft SQL Server 2012 Programming, Indinapolis: John Wiley \& Sons, Inc., 2012, pp. 2. 